\title{
Expression of pY397 FAK promotes the development of non-small cell lung cancer
}

\author{
BAICHUN WANG ${ }^{1,2 *}$, XIUYING QI ${ }^{1 *}$, DANYANG LI $^{3}$, MEIYAN FENG $^{4}$, XIANGNING MENG $^{1}$ and SONGBIN FU ${ }^{1,5}$ \\ ${ }^{1}$ Laboratory of Medical Genetics, Harbin Medical University, Harbin, Heilongjiang 150081; \\ Departments of ${ }^{2}$ Cardiovascular Surgery and ${ }^{3}$ Pathology, The First Affiliated Hospital of Harbin Medical University, Harbin, \\ Heilongjiang 150001; ${ }^{4}$ Department of Pathology, The Third Affiliated Hospital of Harbin Medical University; \\ ${ }^{5}$ Key Laboratory of Medical Genetics, Heilongjiang Higher Education Institutions, Harbin, Heilongjiang 150081, P.R. China
}

Received September 14, 2014; Accepted June 5, 2015

DOI: $10.3892 / \mathrm{ol} .2015 .3992$

\begin{abstract}
Focal adhesion kinase (FAK) expression has been identified as associated with cancer development and metastasis. Autophosphorylation of FAK at tyrosine (Y) 397 (pY397) performs a critical role in tumor cell signaling. However, few studies have evaluated the expression of pY397 FAK in non-small cell lung cancer (NSCLC). In the present study, pY397 FAK expression in NSCLC was investigated using immunohistochemistry. pY397 FAK staining scores were compared between various groups of specimens and the associations between clinical and pathological characteristics were investigated. A Kaplan-Meier survival curve was used to determine the association between pY397 FAK expression and the prognosis of NSCLC patients. The results of the present study revealed that pY397 FAK expression was localized to the cytoplasm of lung cells, and that pY397 FAK was overexpressed in NSCLC tissues, as well as associated metastatic tissues, when compared with the corresponding non-tumor tissues. However, no significant difference was identified between the pY397 FAK expression in primary lesions and lymph node metastases. Furthermore, pY397 FAK staining scores were not found to be associated with the tumor size, gender, degree of differentiation, histotypes, presence of lymph node metastases or survival rate of NSCLC patients. These results indicate that pY397 FAK is
\end{abstract}

Correspondence to: Dr Songbin Fu or Dr Xiangning Meng, Laboratory of Medical Genetics, Harbin Medical University, 157 Baojian Road, Nangang, Harbin, Heilongjiang 150081, P.R. China

E-mail: fusb@ems.hrbmu.edu.cn

E-mail: mengxiangn@ems.hrbmu.edu.cn

*Contributed equally

Abbreviations: FAK, focal adhesion kinase; NSCLC, non-small cell lung cancer

Key words: pY397 focal adhesion kinase, immunohistochemistry, prognosis, non-small cell lung cancer involved with the development of NSCLC, but is not a prognostic marker for the disease.

\section{Introduction}

Focal adhesion kinase (FAK), a $125 \mathrm{kDa}$ cytoplasmic tyrosine (Y) kinase that is localized in focal contacts, is hypothesized to perform a crucial role in the control of integrin-mediated cellular functions, such as cell invasion, migration, cycle progression and survival (1-3). Autophosphorylation of FAK on Y397 (pY397), the most important autophosphorylated site of FAK (4), leads to the activation of FAK and the formation of a multiprotein signaling complex containing FAK at the core (5). Previous reports have revealed the overexpression of FAK in numerous solid cancers (6-12) and metastatic tumors (13-15). Subsequent studies have also revealed that FAK and phosphorylated FAK (p-FAK) are involved in the carcinogenesis of cancers of the digestive organs $(14,16)$. In particular, FAK and p-FAK (pY397 and pY407) expression levels have been found to be closely associated with the differentiation of human colon cancers (17). In addition, Owen et al (18) demonstrated the importance of pY397 FAK signaling in the promotion of cell spreading and migration, suggesting that the interaction between FAK and effector molecules, which bind to this site presents a key step in these processes (18-20). As a result, it was hypothesized that pY397 FAK exhibits an extremely important role during cancer progression.

To the best of our knowledge, only a small number of studies investigating the expression of pY397 FAK in cancer have been performed (17,21-23) and no studies investigating the expression of pY397 FAK in non-small cell lung cancer (NSCLC) have been published in the literature. Therefore, the aim of the present study was to analyze the expression of pY397 FAK in NSCLC to investigate the association between pY397 FAK and the development and prognosis of NSCLC.

\section{Materials and methods}

Ethics statement. The present study was approved by the Institutional Research Board of Harbin Medical University 
(Harbin, Heilongjiang, China). Samples were obtained with informed consent and written informed consent was obtained from each patient.

Tissue specimens.Paraffin-embedded surgical tissue specimens were obtained from 145 patients with NSCLC who underwent curative surgery between January 2001 and October 2005 at The First Affiliated Hospital of Harbin Medical University (Harbin, Heilongjiang, China). Samples were obtained from 145 primary tumor tissues, 87 corresponding non-tumor tissues and 37 lymph node metastases. None of the patients had received radiotherapy or chemotherapy prior to surgical treatment. Representative tissue sections were used for immunohistochemical analysis, and the NSCLC diagnosis for each lesion was pathologically confirmed. The clinicopathological data of the patients are reported in Table I.

Immunohistochemical studies. Immunohistochemical analysis of the paraffin-embedded sections was performed using the PV-9000 kit (Beijing Zhongshan Golden Bridge Biotechnology Co., Ltd., Beijing, China) according to the manufacturer's instructions. For antigen retrieval, the sections were autoclaved for $10 \mathrm{~min}$ at $121^{\circ} \mathrm{C}$. The sections were then incubated with rabbit polyclonal anti-pY397 FAK primary antibodies (cat no. 07-012; Upstate Biotechnology, Inc., Lake Placid, NY, USA) at a dilution of 1:200 at $4^{\circ} \mathrm{C}$ overnight. Two board-certified pathologists scored each tissue section for pY397 FAK expression based on a scoring system $(24,25)$ that measured staining intensity as follows: 0 , none; 1 , borderline; 2 , weak; 3 , moderate; and 4 , strong. The percentage of positive cells (1-100\%), cellular localization (cytoplasm, nucleus, membrane or a combination) and overall distribution (homogeneous or heterogeneous) were also assessed. The expression of pY397 FAK was considered to be strong if the tissue sections exhibited a staining intensity of $3-4$ and $\geq 90 \%$ of the cells exhibited positive immunoreactivity, whereas tissue sections exhibiting a staining score of 0-2 with $<90 \%$ of cells exhibiting positive immunoreactivity were considered to exhibit weak pY397 FAK expression.

Statistical analysis. All statistical analyses were performed using SPSS version 17.0 statistical software (SPSS, Inc., Chicago, IL,USA). The $\chi^{2}$ test was used to compare the intensity of pY397 FAK immunostaining in the non-tumor lung tissues, primary tumors and the corresponding metastatic tissues. In addition, the association between clinicopathological factors and pY397 FAK expression was analyzed using the $\chi^{2}$ test. A survival curve was calculated using the Kaplan-Meier product limit estimate, and differences between patient survival times were analyzed using the log-rank method. $\mathrm{P}<0.05$ was considered to indicate a statistically significant difference.

\section{Results}

pY397 FAK overexpression in primary NSCLC tumors and corresponding lymph node metastases. To confirm whether pY397 FAK was involved in the tumorigenesis of NSCLC, immunohistochemical analyses were conducted to analyze pY397 FAK expression in paraffin-embedded specimens obtained from 145 primary carcinomas, 87 corresponding non-tumor lung tissues and 37 lymph node metastases.
Cytoplasmic pY397 FAK expression was observed in NSCLC and non-tumor lung cells. In non-tumor cells, pY397 FAK protein expression was mainly identified in the normal ciliated columnar epithelium of the bronchus, particularly the apical cilia and the basal surface, as well as in the type 2 alveolar epithelial cells (Fig. 1A). pY397 FAK expression was also identified in non-tumor cells, indicating that FAK autophosphorylation is required for the normal development of cells (26).

In the matched samples, a total of $86(59 \%)$ tumor tissues and $21(57 \%)$ lymph node metastases exhibited strong pY397 FAK expression, while 87 (100\%) non-tumor tissues revealed weak immunostaining (Table II; Fig. 1). From these results, a strong level of pY397 FAK expression was identified in primary NSCLC tumors and the associated lymph node metastases compared with non-tumor tissues $(\mathrm{P}<0.001)$. No significant difference was identified between the pY397 FAK expression levels of the primary tumors and the associated lymph node metastases $(\mathrm{P}=0.778)$. Notably, the expression of pY397 FAK was increased in precancerous lesions and in situ primary carcinoma lesions compared with normal epithelia (Fig. 1). These results indicate that pY397 FAK is involved in tumorigenesis, particularly the early development of NSCLC.

Association between pY397 FAK expression and clinicopathological features of NSCLC patients. To investigate the clinical significance of pY397 FAK in NSCLC, the clinicopathological profiles of patients with and without pY397 FAK overexpression were analyzed, as reported in Table I. No significant differences were identified between pY397 FAK overexpression and patient age, gender, tumor size, differentiation, histotype or lymph node metastasis $(\mathrm{P}>0.05)$. In addition, although no significant difference was identified between the five-year recurrence-free survival rate of patients with and without pY397 FAK overexpression $(\mathrm{P}>0.05)$, patients with strong pY397 FAK expression exhibited a trend towards shorter survival time (Fig. 2). These results indicate that pY397 FAK overexpression is not a prognostic marker in NSCLC.

\section{Discussion}

FAK, which is activated by phosphorylation of Y397, regulates numerous cellular functions and is critical for cancer progression. FAK overexpression has been observed in various cancers (26). However, to the best of our knowledge, only a small number of studies have demonstrated an association between FAK phosphorylation on pY397 and cancer using human tissue samples $(17,21-23)$.

In the present study, pY397 FAK expression in NSCLC tissues was analyzed. Strong pY397 FAK expression was identified in the majority of primary cancer tissue samples, as well as metastatic lymph nodes, whereas weak pY397 FAK expression was identified in non-tumor lung tissues. In addition, no significant difference in pY397 FAK expression was identified between primary lesions and lymph node metastases. However, increased pY397 FAK expression was observed in precancerous lesions and in situ primary carcinoma lesions compared with the expression in normal epithelia. These results are consistent with those of previous studies. For example, 
Table I. Associations between pY397 FAK expression and the clinical and pathological characteristics of 145 non-small cell lung cancer patients.

\begin{tabular}{|c|c|c|c|c|}
\hline \multirow[b]{2}{*}{ Parameter } & \multirow[b]{2}{*}{$\mathrm{n}$} & \multicolumn{2}{|c|}{ pY397 FAK expression } & \multirow[b]{2}{*}{ P-value } \\
\hline & & Weak, $\mathrm{n}$ & Strong, $\mathrm{n}$ & \\
\hline Age & & & & 0.405 \\
\hline$\leq 60$ years & 80 & 35 & 45 & \\
\hline$>60$ years & 65 & 24 & 41 & \\
\hline Gender & & & & 0.133 \\
\hline Male & 94 & 34 & 60 & \\
\hline Female & 51 & 25 & 26 & \\
\hline Size & & & & 0.704 \\
\hline$<3 \mathrm{~cm}$ & 49 & 21 & 28 & \\
\hline$\geq 3 \mathrm{~cm}$ & 96 & 38 & 58 & \\
\hline \multicolumn{5}{|l|}{ Differentiation } \\
\hline Well-moderate & 91 & 41 & 50 & 0.165 \\
\hline Low & 54 & 18 & 36 & \\
\hline Histotype & & & & 0.754 \\
\hline Adenocarcinoma & 69 & 29 & 40 & \\
\hline Squamous cell carcinoma & 76 & 30 & 46 & \\
\hline Lymph node metastasis & & & & 0.764 \\
\hline Present & 71 & 28 & 43 & \\
\hline Absent & 74 & 31 & 43 & \\
\hline
\end{tabular}

FAK, focal adhesion kinase; Y, tyrosine; pY397, phosphorylated Y397.

Table II. Expression of pY397 FAK in non-tumor tissues, primary NSCLC tissue and lymph node metastases.

\begin{tabular}{lrccr}
\hline & & \multicolumn{2}{c}{ pY397 FAK expression } & \\
\cline { 3 - 4 } Tissue & $\mathrm{n}$ & Weak, $\mathrm{n}(\%)$ & Strong, $\mathrm{n}(\%)$ & P-value \\
\hline Non-tumor & 87 & $87(100)$ & $0(0)$ & $0.000^{\mathrm{a}}$ \\
Primary NSCLC & 145 & $59(41)$ & $86(59)$ & $0.778^{\mathrm{b}}$ \\
Lymph node metastases & 37 & $16(43)$ & $21(57)$ &
\end{tabular}

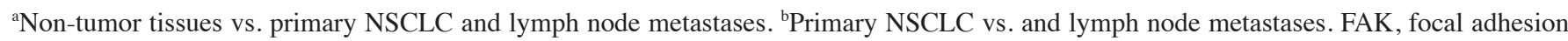
kinase; Y, tyrosine; pY397, phosphorylated Y397; NSCLC, non-small cell lung cancer.

Madan et al (22) observed no pY397 FAK immunostaining in benign epithelium samples, while strong pY397 FAK staining was observed in all breast carcinoma tissues analyzed. Furthermore, Grisaru-Granovsky et al (27) observed high levels of pY397 FAK expression in invasive ovarian samples, but not in normal ovarian epithelium and Aronsohn et al (28) found that pY397 FAK was overexpressed in laryngeal squamous carcinoma tissues compared with normal squamous epithelium. Madan et al (22) also demonstrated that pY397 FAK overexpression was found to be correlated with malignant transformation, but not the development of invasive tumor properties. By contrast, Carelli et al (29) performed western blot analysis, which demonstrated that pY397 FAK was equally phosphorylated in the neoplastic and non-neoplastic regions of lung cancer tissue. However, the present study hypothesizes that the tissue homogenates analyzed in the study by Carelli et al (29) may have contained a mixture of epithelium and surrounding stromal contaminants. In addition, the sample size used was relatively small. Consequently, these two factors may have affected the expression of pY397 FAK observed in NSCLC tissues. In the present study, the data was obtained from the analysis of 145 NSCLC samples by immunohistochemistry, which may be used to stain the epithelium specifically. The results of the present study indicate that pY397 FAK is involved in the process of malignant transformation, particularly the early stages. However, pY397 FAK was not found to correlate with the metastatic phenotype in NSCLC. 
A

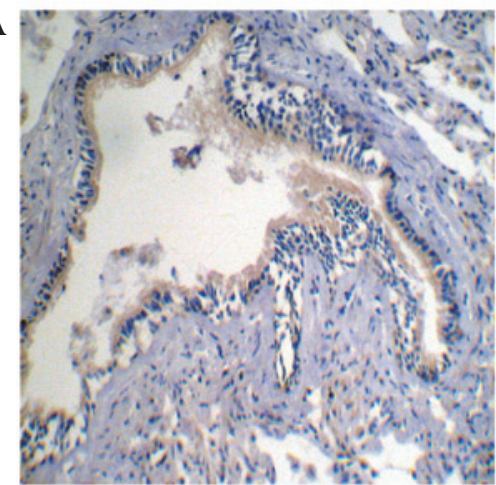

C

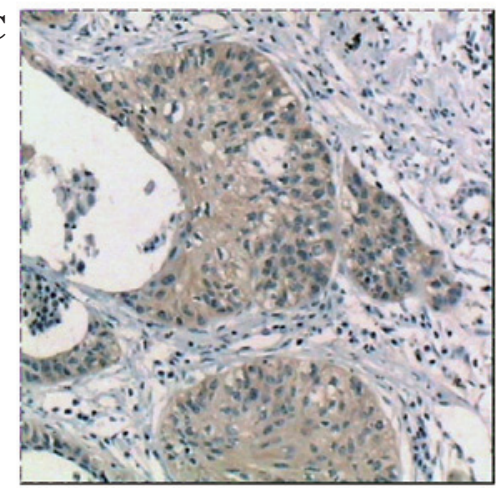

E

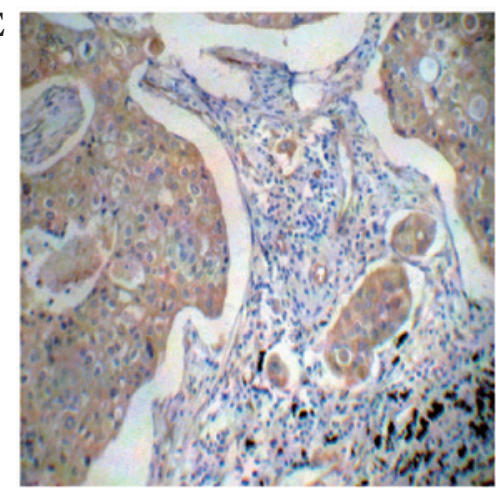

B

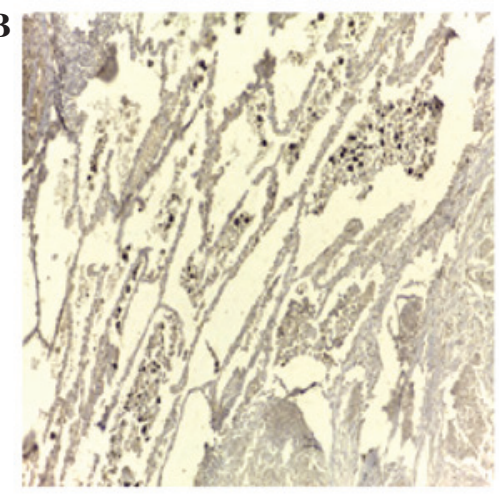

D

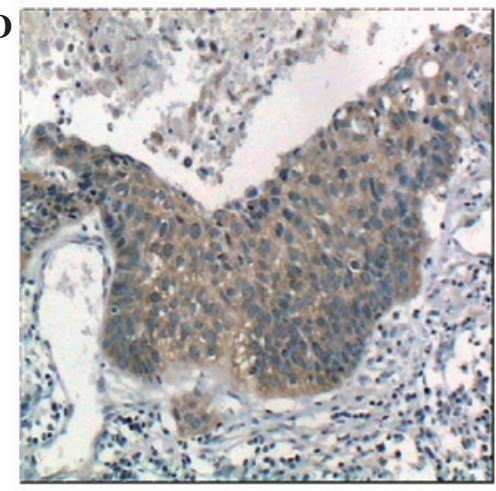

F

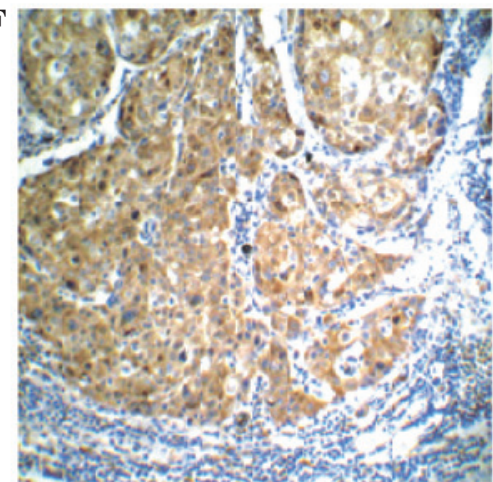

Figure 1. Immunohistochemical analysis of pY397 FAK staining in non-small cell lung cancer and non-tumor tissues using a DM750 microscope (Leica Microsystems GmbH, Wetzlar, Germany). Weak pY397 FAK staining was observed in (A) normal ciliated columnar epithelium of the bronchus and (B) alveolar epithelial cells, while strong pY397 FAK staining was identified in (C) precancerous lesions, (D) carcinoma in situ, (E) primary lesions and (F) lymph node metastases (magnification, x100). DAB staining. FAK, focal adhesion kinase; Y, tyrosine; pY397, phosphorylated Y397.

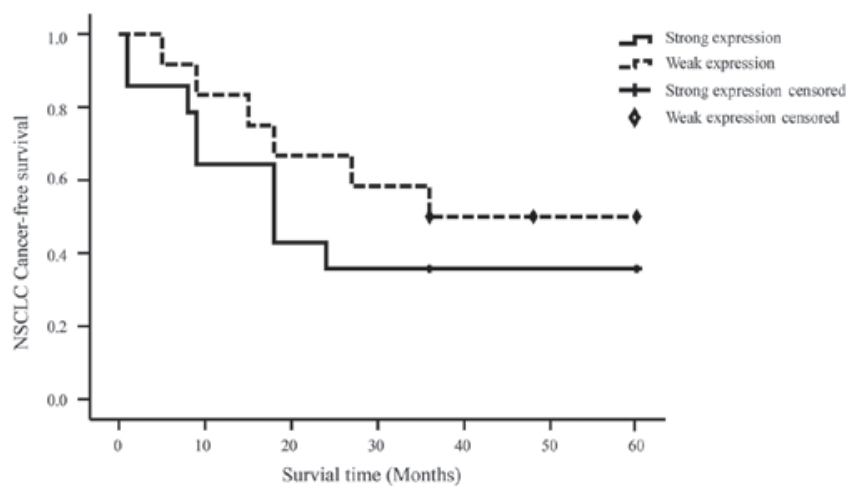

Figure 2. The association between pY397 FAK expression levels and survival rate of NSCLC patients. Kaplan-Meier analysis revealed that patients with strong pY397 FAK expression exhibited a trend towards a lower five-year recurrence-free survival rate compared with patients with weak expression. However, no significant difference was identified $(\mathrm{P}>0.05)$. FAK, focal adhesion kinase; Y, tyrosine; pY397, phosphorylated Y397; NSCLC, non-small cell lung cancer.
The upregulation of pY397 FAK observed in primary and precancerous lesions indicates that the overexpression of pY397 FAK may be a feature of early stage NSCLC cells. The inhibition of pY397 FAK expression of breast cancer and oral squamous cell carcinoma by TAE226, a FAK inhibitor, was found to significantly suppress the proliferation and caspase-mediated apoptosis $(30,31)$, indicating that pY397 FAK may contribute to the malignant transformation of NSCLC by promoting proliferation and suppressing apoptosis. Compared with primary lesions, the lack of change in pY397 FAK expression in the metastatic lymph nodes may predict a completely anchorage-independent phenotype in additional tumor progression, or the lack of change in expression may suggest that certain signals are no longer required while other signals emerge. For example, Slack et al (32) demonstrated that increased FAK expression in combination with pY861 FAK contributed to an increase in the cell motility of prostate cancer cells. However, further studies are required to investigate these findings. 
Additionally, in the present study, no association was identified between pY397 FAK expression and clinicopathological factors, including the presence of lymph node metastases and the survival rate, suggesting that pY397 FAK is not a prognostic marker for NSCLC.

In conclusion, the results of the present study indicate that the overexpression of pY397 FAK during NSCLC progression may be involved with the development and maintenance of NSCLC. pY397 FAK positivity does not predict patient outcome, however, its expression may serve as an attractive therapeutic target. In addition, the mechanism of overexpression of pY397 FAK in NSCLC has yet to be clarified.

\section{Acknowledgements}

This study was supported by the International Science and Technology Cooperation Program of China (grant no. 2013DFA31610) and the Natural Science Foundation in Heilongjiang Province of China (grant no. QC08C90).

\section{References}

1. Schaller MD, Borgman CA, Cobb BS, Vines RR, Reynolds AB and Parsons JT: pp125FAK a structurally distinctive protein-tyrosine kinase associated with focal adhesions. Proc Natl Acad Sci USA 89: 5192-5196, 1992.

2. van Nimwegen MJ and van de Water B: Focal adhesion kinase: A potential target in cancer therapy. Biochem Pharmacol 73: 597-609, 2007.

3. Megison ML, Stewart JE, Nabers HC, Gillory LA and Beierle EA: FAK inhibition decreases cell invasion, migration and metastasis in MYCN amplified neuroblastoma. Clin Exp Metastasis 30: 555-568, 2013.

4. Schlaepfer DD, Mitra SK and Ilic D: Control of motile and invasive cell phenotypes by focal adhesion kinase. Biochim Biophys Acta 1692: 77-102, 2004.

5. Schaller MD: Biochemical signals and biological responses elicited by the focal adhesion kinase. Biochim Biophys Acta 1540: 1-21, 2001

6. Oktay MH, Oktay K, Hamele-Bena D, Buyuk A and Koss LG: Focal adhesion kinase as a marker of malignant phenotype in breast and cervical carcinomas. Hum Pathol 34: 240-245, 2003.

7. Watermann DO, Gabriel B, Jäger M, Orlowska-Volk M, Hasenburg A, zur Hausen A, Gitsch G and Stickeler E: Specific induction of pp125 focal adhesion kinase in human breast cancer. Br J Cancer 93: 694-698, 2005.

8. Rovin JD, Frierson HF Jr, Ledinh W, Parsons JT and Adams RB: Expression of focal adhesion kinase in normal and pathologic human prostate tissues. Prostate 53: 124-132, 2002.

9. Schneider GB, Kurago Z, Zaharias R, Gruman LM, Schaller MD and Hendrix MJ: Elevated focal adhesion kinase expression facilitates oral tumor cell invasion. Cancer 95: 2508-2515, 2002.

10. Judson PL, He X, Cance WG and Van Le L: Overexpression of focal adhesion kinase, a protein tyrosine kinase, in ovarian carcinoma. Cancer 86: 1551-1556, 1999.

11. Kim SJ, Park JW, Yoon JS, et al: Increased expression of focal adhesion kinase in thyroid cancer: Immunohistochemical study. J Korean Med Sci 19: 710-715, 2004.

12. Fujii T, Koshikawa K, Nomoto S, Okochi O, Kaneko T, Inoue S, Yatabe Y, Takeda S and Nakao A: Focal adhesion kinase is overexpressed in hepatocellular carcinoma and can be served as an independent prognostic factor. J Hepatol 41: 104-111, 2004.

13. Lark AL, Livasy CA, Calvo B, Caskey L, Moore DT, Yang X and Cance WG: Overexpression of focal adhesion kinase in primary colorectal carcinomas and colorectal liver metastases: Immunohistochemistry and real-time PCR analyses. Clin Cancer Res 9: 215-222, 2003.

14. Cance WG, Harris JE, Iacocca MV, et al: Immunohistochemical analyses of focal adhesion kinase expression in benign and malignant human breast and colon tissues: Correlation with preinvasive and invasive phenotypes. Clin Cancer Res 6: 2417-2423, 2000.
15. Miyazaki T, Kato H, Nakajima M, Sohda M, Fukai Y, Masuda N, Manda R, Fukuchi M, Tsukada K and Kuwano H: FAK overexpression is correlated with tumour invasiveness and lymph node metastasis in oesophageal squamous cell carcinoma. Br J Cancer 89: 140-145, 2003.

16. Murata T, Naomoto Y, Yamatsuji T, et al: Localization of FAK is related with colorectal carcinogenesis. Int J Oncol 32: 791-796, 2008.

17. Matkowskyj KA, Keller K, Glover S, Kornberg L, Tran-Son-Tay R and Benya RV: Expression of GRP and its receptor in well-differentiated colon cancer cells correlates with the presence of focal adhesion kinase phosphorylated at tyrosines 397 and 407. J Histochem Cytochem 51: 1041-1048, 2003.

18. Owen JD, Ruest PJ, Fry DW and Hanks SK: Induced focal adhesion kinase (FAK) expression in FAK-null cells enhances cell spreading and migration requiring both auto- and activation loop phosphorylation sites and inhibits adhesion-dependent tyrosine phosphorylation of Pyk2. Mol Cell Biol 19: 4806-4818, 1999.

19. Cary LA, Chang JF and Guan JL: Stimulation of cell migration by overexpression of focal adhesion kinase and its association with Src and Fyn. J Cell Sci 109: 1787-1794, 1996.

20. Richardson A, Malik RK, Hildebrand JD and Parsons JT: Inhibition of cell spreading by expression of the C-terminal domain of focal adhesion kinase (FAK) is rescued by coexpression of Src or catalytically inactive FAK: A role for paxillin tyrosine phosphorylation. Mol Cell Biol 17: 6906-6914, 1997.

21. Fan DM and Shi HR: Pilot study: Alteration of deleted in liver cancer 1 and phosphorylated focal adhesion kinase Y397 cytoplasmic expression and the prognostic value in advanced epithelial ovarian carcinoma. Int J Mol Sci 12: 8489-8501, 2011.

22. Madan R, Smolkin MB, Cocker R, Fayyad R and Oktay MH: Focal adhesion proteins as markers of malignant transformation and prognostic indicators in breast carcinoma. Hum Pathol 37: 9-15, 2006

23. Lai IR, Chu PY, Lin HS, Liou JY, Jan YJ, Lee JC and Shen TL: Phosphorylation of focal adhesion kinase at Tyr397 in gastric carcinomas and its clinical significance. Am J Pathol 177: 1629-1637, 2010

24. Lark AL, Livasy CA, Dressler L, et al: High focal adhesion kinase expression in invasive breast carcinomas is associated with an aggressive phenotype. Mod Pathol 18: 1289-1294, 2005

25. Lightfoot HM Jr, Lark A, Livasy CA, Moore DT, Cowan D, Dressler L, Craven RJ and Cance WG: Upregulation of focal adhesion kinase (FAK) expression in ductal carcinoma in situ (DCIS) is an early event in breast tumorigenesis. Breast Cancer Res Treat 88: 109-116, 2004.

26. CorsiJM,HoubronC,BilluartP,etal:Autophosphorylation-independent and-dependent functions of focal adhesion kinase during development. J Biol Chem 284: 34769-34776, 2009.

27. Grisaru-Granovsky S, Salah Z, Maoz M, Pruss D, Beller U and Bar-Shavit R: Differential expression of protease activated receptor 1 (Par1) and pY397FAK in benign and malignant human ovarian tissue samples. Int J Cancer 113: 372-378, 2005.

28. Aronsohn MS, Brown HM, Hauptman G and Kornberg LJ: Expression of focal adhesion kinase and phosphorylated focal adhesion kinase in squamous cell carcinoma of the larynx. Laryngoscope 113: 1944-1948, 2003.

29. Carelli S, Zadra G, Vaira V, et al: Up-regulation of focal adhesion kinase in non-small cell lung cancer. Lung Cancer 53: 263-271, 2006.

30. Kurio N, Shimo T, Fukazawa T, Takaoka M, Okui T, Hassan NM, Honami T, Hatakeyama S, Ikeda M, Naomoto Y, et al: Anti-tumor effect in human breast cancer by TAE226, a dual inhibitor for FAK and IGF-IR in vitro and in vivo. Exp Cell Res 317: 1134-1146, 2011

31. Kurio N, Shimo T, Fukazawa T, et al: Anti-tumor effect of a novel FAK inhibitor TAE226 against human oral squamous cell carcinoma. Oral Oncol 48: 1159-1170, 2012.

32. Slack JK, Adams RB, Rovin JD, Bissonette EA, Stoker CE and Parsons JT: Alterations in the focal adhesion kinase/Src signal transduction pathway correlate with increased migratory capacity of prostate carcinoma cells. Oncogene 20: 1152-1163, 2001. 